\title{
Minimising clearance consumption: a key factor for the design of blades robust to rotor/stator interactions ?
}

\author{
A. Batailly ${ }^{1}$, A. Millecamps ${ }^{2}$
}

\begin{abstract}
The recent development of a numerical strategy dedicated to the simulation of rotor/stator interactions stemming from structural contacts in modern aircraft engines led to the first optimization of a high-pressure compressor blade profile accounting for criteria related to nonlinear contact simulations. This optimization procedure revealed very significant improvements in terms of amplitudes of vibration but failed to identify key design parameters. Satisfying numerical results were obtained by a minor modification of a combination of many design parameters. Based on this observation, this contribution intends to shed a new light on this previous redesign operation focusing on one key quantity: the clearance consumption. This quantity is presented in the first section. In the second section, results of the redesign operation are recalled before the presentation of original results, featuring detailed interaction maps in the frequency domain, on which focuses the third section of the article. Finally, the blade profiles are extensively compared based on their specific clearance consumption and presented results suggest that this quantity may be key in discriminating acceptable from unacceptable blade profiles with respect to their vibratory behaviour when structural contacts occur.

\section{Keywords}

rotor/stator interaction; rubbing; nonlinear dynamics; unilateral contact dynamics; friction; abradable coating; clearance consumption

1 - Department of Mechanical Engineering, École Polytechnique de Montréal, P.O. Box 6079, Succ. Centre-Ville, Montréal, Québec, Canada H3C 3A7

2 - Safran Aircraft Engines, site de Villaroche, Moissy-Cramayel, 77550, France
\end{abstract}




\title{
Minimisation de la consommation de jeu : un facteur clé pour la conception d'aubes robustes aux interactions rotor/stator?
}

\author{
A. Batailly ${ }^{1}$, A. Millecamps ${ }^{2}$
}

\begin{abstract}
Résumé
Le développement récent d'un outil numérique dédié à la simulation d'interactions rotor/stator engendrées par des contacts dans les moteurs d'avion a permis de mener à bien la première optimisation d'une aube de compresseur haute-pression en prenant en compte des critères issus de la dynamique non-linéaire. Cette procédure d'optimisation a notamment mis en lumière des gains très significatifs en termes d'amplitudes de la réponse vibratoire de l'aube optimisée par rapport à l'aube initiale. En revanche, elle n'a pas permis d'identifier les paramètres de conception clés pour obtenir de tels résultats. L'aube optimisée a été obtenue en modifiant légèrement une combinaison de plusieurs paramètres de conception. Prenant acte de cette observation, cet article propose d'observer sous un autre angle les résultats précédemment obtenus et se focalise sur une quantité particulière : la consommation de jeu. La première section de l'article présente en détails cette quantité. Dans la section suivante, les résultats de la procédure d'optimisation sont brièvement rappelés et complétés par de nouveaux résultats, incluant des cartes d'interaction détaillées dans le domaine fréquentiel. Dans la dernière section, les profils d'aubes obtenus au cours de la procédure d'optimisation sont comparés relativement à leur consommation de jeu et les résultats présentés suggèrent que cette quantité pourrait permettre de discriminer les profils d'aubes acceptables de ceux qui ne le sont pas relativement à leur amplitude de vibration lorsque des contacts aubes/carter se produisent.

\section{Mots-clés}

interaction rotor/stator; frottement aube/carter; dynamique non-linéaire; dynamique du contact; frottement; revêtement abradable; consommation de jeu

1 - Département de génie mécanique, École Polytechnique de Montréal, P.O. Box 6079, Succ. Centre-Ville, Montréal, Québec, Canada H3C 3A7

2 - Safran Aircraft Engines, site de Villaroche, Moissy-Cramayel, 77550, France
\end{abstract}




\section{NOMENCLATURE}

amplitude of the blade oscillation

$c_{c} \quad$ clearance consumption

1B first bending mode

$1 \mathrm{~T} \quad$ first torsion mode

LE leading edge

MC middle of chord

TE trailing edge

\section{INTRODUCTION}

This contribution intends to bring new insight regarding the redesign operation of a highpressure compressor blade within an aircraft engine previously published by the same authors [1]. This redesign was carried out on an industrial blade profile which was found sensitive to rotor/stator interactions stemming from blade-tip/casing structural contacts. Such interactions are of particular interest for aircraft engine manufacturers. In fact, an engine efficiency is intimately related to the reduction of parasitic leakages from a stage of the engine to another. Such reduction may only be achieved through a closing of operating blade-tip/casing clearances which leads to more frequent structural contacts that may initiate the aforementioned interaction phenomena. In this context, the development of blades and bladed components robust to structural contacts is a growing field of investigation and motivated many recent numerical investigations $[2,3,4]$.

The redesign operation that was previously carried out introduced a few optimization criteria [1] related to non-linear structural simulations between the blade of interest and a perfectly rigid casing on which was deposited an abradable coating. It led to an optimized blade profile that was shown, from a numerical standpoint, to be numerically insensitive to the investigated contact scenarios. The improvement in terms of the blade vibration amplitudes following structural contacts were made without impacting aerodynamic performances and the additional mass was limited to $+4.15 \%$. Also, the impact on the blade first natural frequencies was limited to a few percents while the maximum Von Mises stress due to centrifugal effects was reduced by $31.90 \%$. In fact, it seems that typical design parameters underwent relatively small variations while amplitudes of vibration significantly dropped. Thus, there is a need for further analyses of this redesign operation in order to identify possibly key design factors for bladed components. In order to do so, this paper focuses on one particular quantity: the clearance consumption along the blade tip.

Studies focusing on blade-tip/casing clearances usually focus solely on aerodynamic performances $[5,6,7]$. As structural interactions become of prime importance for aircraft engine manufacturers, there is a need for the convergence of the analyses carried out from aerodynamic and structural standpoints. As an example, recent optimization studies conducted from both an aerodynamic standpoint [8] and a structural standpoint [1] led to opposite conclusions: the former suggests that clearance closure as the blade vibrates increases aerodynamic performances while the latter underline that clearance closure should be avoided because of its negative consequences when structural contacts occur. In this context, this paper aims at introducing clearance consumption as a possibly relevant quantity for the optimization of blade profiles. Details regarding the computation of this quantity are given in the first section of the paper. In the second section, a brief summary of the redesign operation carried out in [1] is proposed. Then, in the third section, new results obtained from numerical simulations with the optimized blade profiles are provided. These results underline the distinct vibration behaviour of the iterated profiles. Finally, an in-depth comparison of clearance consumptions for each iterated profile is provided in the fourth section before the presentation of a case study for non-divergent interactions. 


\section{CLEARANCE CONSUMPTION: DEFINITION}

Clearance consumption, noted $c_{c}$, is a quantity that refers to the evolution of the bladetip/abradable coating clearance as the blade vibrates. The abradable coating, deposited along the casing circumference, may be worn out after being impacted by the blade which yield a local increase of clearances. This wear phenomenon is accounted for in the employed numerical strategy. The clearance consumption for the investigated high-pressure compressor blade will be scrutinized with an emphasis on its first bending (1B) and torsion (1T) modes. It will be computed in three locations: the leading edge (LE), the middle of chord (MC) and the trailing edge (TE). Exemplarily, Fig. 1 pictures the clearance consumption of a blade

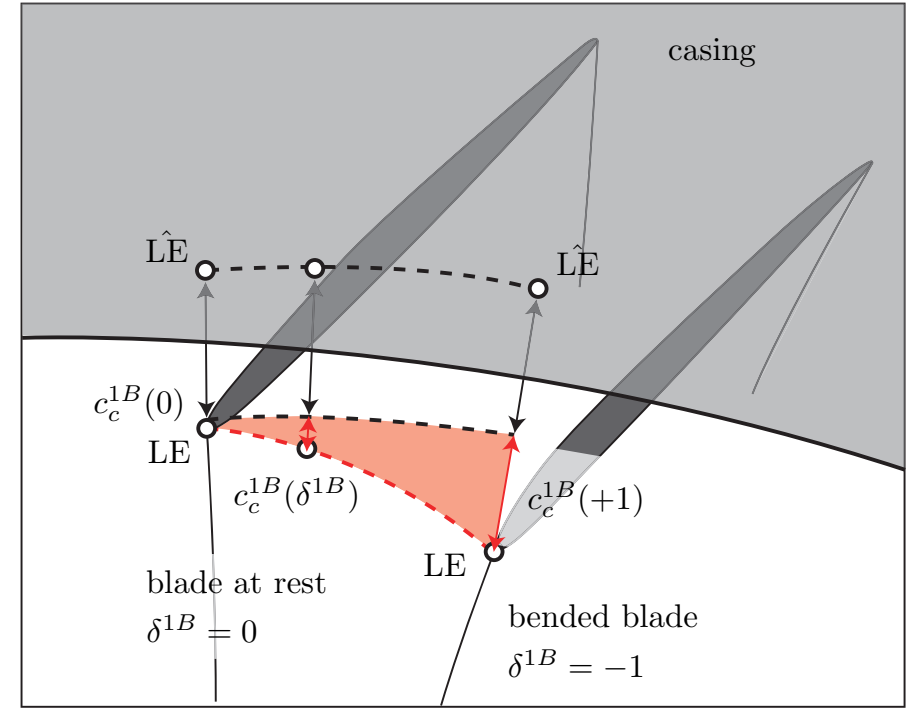

Figure 1. VISUAL REPRESENTATION OF THE CLEARANCE CONSUMPTION

being deformed along its first bending mode. The distance between the blade leading edge (LE) and its closest counterpart on the contact surface of the abradable coating, denoted $\mathrm{LE}$, is the sum of the blade/casing clearance at rest $(\longleftrightarrow)$ with the clearance consumption $c_{c}(\longleftrightarrow)$. For the computation of $c_{c}$ possible contacts between the blade and the abradable coating are not considered: only the blade is accounted for. The following convention is adopted:

when $\mathbf{c}_{\mathbf{c}}<\mathbf{0}$ the clearance between the blade and the coating is increased (this corresponds to the configuration pictured in Fig. 1),

when $\mathbf{c}_{\mathbf{c}}>\mathbf{0}$ the clearance between the blade and the coating is decreased.

The value of $c_{c}$ is looked at over a certain amplitude of deformation $\delta \in\left[\delta_{\min } ; \delta_{\max }\right]$ for both modes $1 \mathrm{~B}$ and $1 \mathrm{~T}$, using the following criterion:

$\left\|\mathbf{u}_{\mathrm{LE}, r}^{M}\right\| \leq 0.25 \mathrm{~mm}$

where $\mathbf{u}_{\mathrm{LE}, r}^{M}$ is the radial displacement of the blade leading edge when the blade vibrates along its free-vibration mode $M$. The following notations are used in the paper:

$$
\begin{aligned}
& \delta_{\min }^{1 \mathrm{~B}}=-1 \Leftrightarrow \mathbf{u}_{\mathrm{LE}, r}^{1 B}=-0.25 \mathrm{~mm} \\
& \delta_{\max }^{1 \mathrm{~B}}=+1 \Leftrightarrow \mathbf{u}_{\mathrm{LE}, r}^{1 B}=0.25 \mathrm{~mm} \\
& \delta_{\min }^{1 \mathrm{~T}}=-1 \Leftrightarrow \mathbf{u}_{\mathrm{LE}, r}^{1 T}=-0.25 \mathrm{~mm} \\
& \delta_{\max }^{1 \mathrm{~T}}=+1 \Leftrightarrow \mathbf{u}_{\mathrm{LE}, r}^{1 T}=0.25 \mathrm{~mm}
\end{aligned}
$$

One may note that the clearance consumption is usually distinct from the blade radial displacement (these quantities may only be equal if the contact surface of the abradable coating is perfectly clylindrical).

\section{RECAPITULATION OF PREVIOUS WORK}




\section{General overview}

This section aims at providing the reader with key elements of the article [1] on which is based the current work. While the text has been reformulated for the sake of brevity, all the details - including the figures - contained in this section have already been published [1]. The blade of a high-pressure compressor underwent structural divergence after blade/abradable coating contact occurrences on a rig test. Thus, there was a need for a re-design operation of this blade in order to avoid potentially high amplitudes of vibration that may significantly shorten its lifespan. The same terminology is used in this article and in [1]: the defective blade is called the initial profile, it is pictured in Fig. 2. Through out the re-design operation, several blade profiles were generated, the focus is made on two of them: the first iterated profile and the final profile, respectively a first iteration providing satisfying results and the final result of the re-design operation. A few key design parameters related to the blade
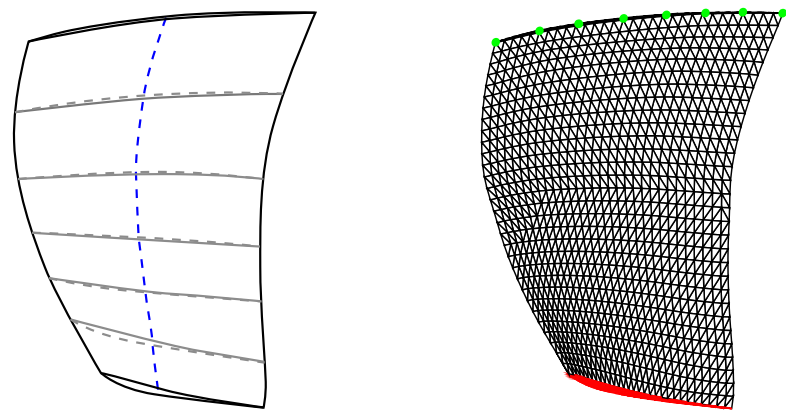

Figure 2. STACKING LAW AND MESH (CLAMPED AREA IN RED, CONTACT NODES IN GREEN) OF THE INITIAL PROFILE

stacking law, see Fig. 2, were identified and used for the re-design operation. The criteria used for optimizing the blade were related to the following quantities:

1. the clearance consumption;

2. the stress field in the blade during an interaction;

3. the behaviour of the vibration amplitudes for an interaction speed.

Very significant differences were obtained between the optimized profiles and the initial profile. As an example, the maximum Von-Mises stress within the blade due to centrifugal stiffening was reduced by almost $32 \%$, see Tab. 1 .

\begin{tabular}{l|rrr}
\hline profile & initial & first iterated & final \\
$\sigma_{c}$ evolution $(\%)$ & 0 & -28.32 & -31.90 \\
\hline
\end{tabular}

Table 1. EVOLUTION OF $\sigma_{c}$ WITHIN THE BLADE PROFILE

\section{Linear and non-linear considerations}

The numerical simulation of blade/casing or blade/abradable coating interactions are highly non-linear since contact areas are a priori unknown and both the contact pressure and the resulting displacements are part of the solution. This non-linearity is accounted for in the employed numerical strategy using a Lagrange-multiplier based algorithm. From a modeling standpoint however, linear finite element models are employed since the considered framework is the one of small perturbations: the focus is made on the arisal of interaction phenomena, where amplitudes of vibration remain small with respect to the charasteristics dimensions of the blade. Thus, the comparison of clearance consumptions between linear and non-linear models presented in section 4.1 intends to give additional insights on the numerical results by assessing up to which amplitude of deformation linear models may accurately capture the clearance consumption of non-linear models.

\section{VIBRATION BEHAVIOR OF ITERATED PROFILES}

The numerical strategy employed for the simulation of rotor/stator interactions has been detailed in previous publications [9] and is not recalled here for the sake of brevity. It relies 
on an explicit time integration procedure [10] combined with a Lagrange multiplier based contact management technique in order to avoid residual penetrations between structures. Convergence with respect to the parameters of the study (time step, reduction parameter of the blade model, density of abradable coating elements along the casing circumference...) has been checked prior to the proposed analysis.

At $t=0 \mathrm{~s}$, the blade is at rest and the casing is perfectly circular. For $t>0 \mathrm{~s}$, the blade angular speed is constant: $\Omega$. Centrifugal stiffening as well as the casing deformation are progressively applied over the first $20 \%$ of the time simulation. The imposed deformation of the casing is the key difference between the distinct contact scenarios considered in the following. Two contact scenarios were considered in [1]: (1) in the first one, the casing deformation is an ovalization featuring two symmetric lobes and (2) in the second scenario, the casing deformation features four symmetric contact areas. Results presented in the frequency domain in this paper complement the ones given in the time domain in [1]. For both contact scenarios, numerical simulations were carried out over a large angular speed range $\Omega \in[0.159 ; 0.3978]$. For each angular speed, a Fourier transform of the leading edge radial displacement over the last 100 revolutions has been performed. The choice of the radial displacement is related to the fact that this quantity may be more representative of the wear level than tangential or axial displacements. Yet, considering a displacement type or another does not impact qualitatively the observations made below. Put side by side, these spectra create an interaction map where amplitudes of vibration are represented using the following colour code: lowest amplitudes of vibration in blue, highest amplitudes of vibration in red. The same colour code is used for all the interaction maps depicted in the remainder.

\section{2-lobe contact scenario}

The comparison between the interaction maps depicted in Figs. 3(a), 3(b) and 3(c) underlines the significant gain in terms of amplitudes of vibration between the initial profile and the iterated profiles. Indeed, the only modification between the carried out simulations is the blade profile. In the context of this article, it is noteworthy that highest amplitudes of vibration, namely the interaction areas, may be located around the modes $1 \mathrm{~B}$ and $1 \mathrm{~T}$ independently one from another, see Fig. 3(a), areas A and B. Thus, interactions may involve mode $1 \mathrm{~B}$ only or mode $1 \mathrm{~T}$ only and may be of different nature. While both $1 \mathrm{~B}$ and $1 \mathrm{~T}$ related interactions vanish with the final profile, $1 \mathrm{~B}$ interactions are still predicted with the first iterated profile as it may be seen in Fig. 3(b), area $\mathbf{C}$.

Repeated structural contacts between the casing and the blade logically lead to a synchronous vibration behaviour: that is why peaks of vibration are essentially located along engine order lines for which the frequency $f$ is a multiple of the angular speed $\Omega$ : $f=k \Omega, k \in \mathbb{N}$. The aforementioned interaction areas are found in the vicinity of an intersection between an engine order line and an eigenfrequency of the blade. However, the highly non-linear nature of the simulated interaction does not allow for an obvious identification of interaction areas: an intersection between an engine order line and an eigenfrequency is not necessarily related to an interaction, see Fig. 3(c).

With this 2-lobe contact scenario, it is underlined that numerical simulations predict that three distinct blade profiles featuring identical aerodynamic performances may lead to extremely different vibration behaviour following structural contacts. It should be recalled here that the contact configuration (incursion depth, thickness of the abradable coating, clearance at rest...) is identical from a profile to another. In order to assess the versatility of the iterated profiles, meaning that the presented results are not limited to a single contact scenario, a 4-lobe contact scenario is considered in the following section.

\section{4-lobe contact scenario}

Interaction maps obtained with a 4-lobe contact scenario confirm previous observations. As a matter of fact, bending or torsion induced interactions are predicted for the initial profile, see Fig. 4(a), areas D and E. Both types of interaction vanish with the final profile in Fig. 4(c) while bending induced interactions are still predicted for the first iterated profile as depicted in Fig. 4(b), see areas $\mathbf{F}$ and G. With a 4-lobe contact scenario, the location of predicted interaction areas matches the location of predicted interaction areas of a 2-lobe 


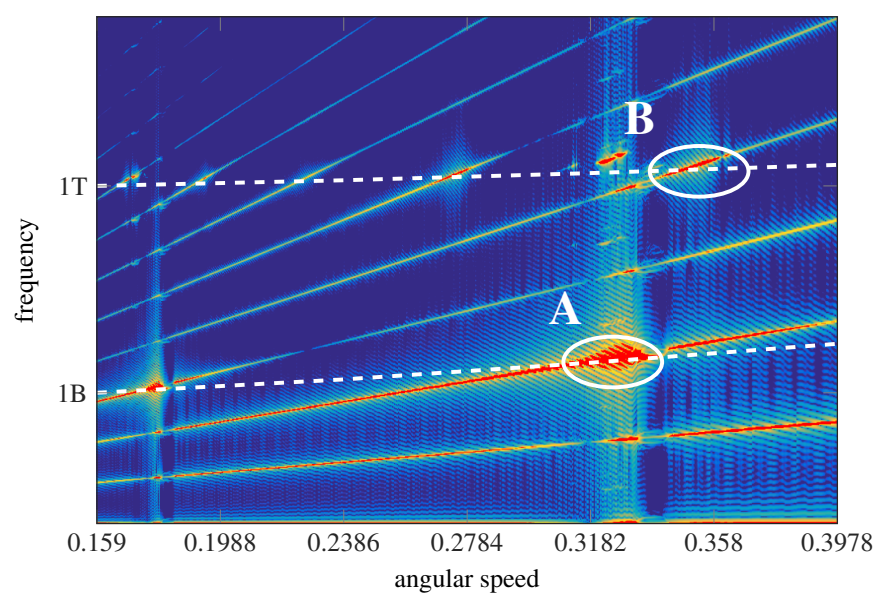

(a) INTIAL PROFILE

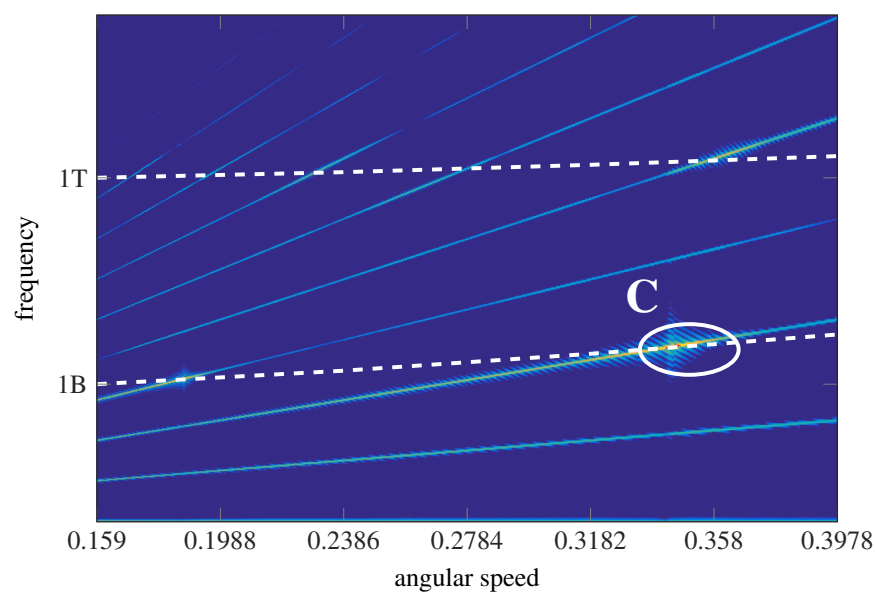

(b) FIRST ITERATED PROFILE

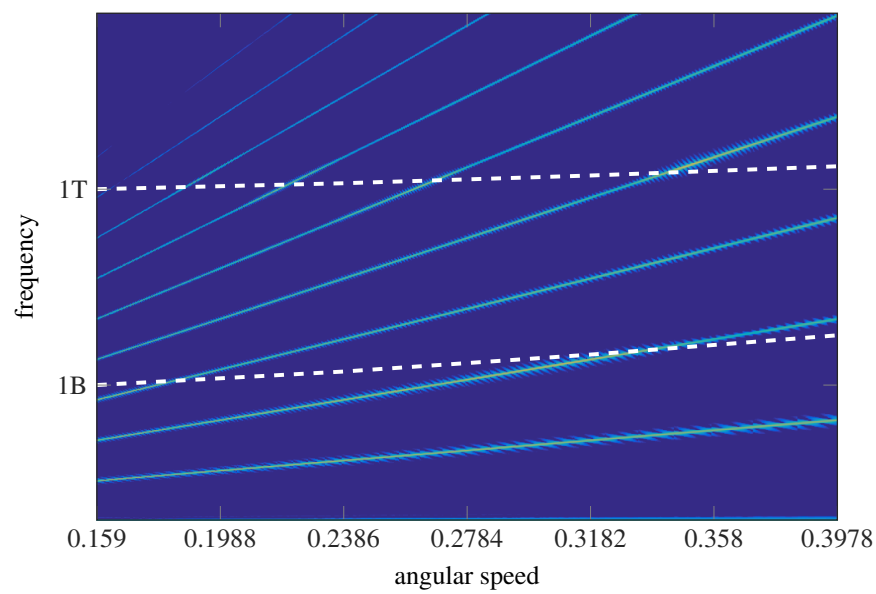

(c) FINAL PROFILE

Figure 3. INTERACTION MAPS WITH 2 LOBES

contact scenario - areas $\mathbf{A}$ and $\mathbf{B}$ in Fig. 3(a) correspond to areas $\mathbf{D}$ and $\mathbf{E}$ in Fig. 4(a) - but they are significantly wider along the horizontal direction (in angular speed) which indicates that the blade profile is more sensitive to this 4-lobe contact scenario.

In any case, the interaction maps drawn for both contact scenarios underline the very significant improvement in terms of amplitudes of vibration between the initial profile and the final (optimized) profile. In the remainder, the focus is made on the clearance 


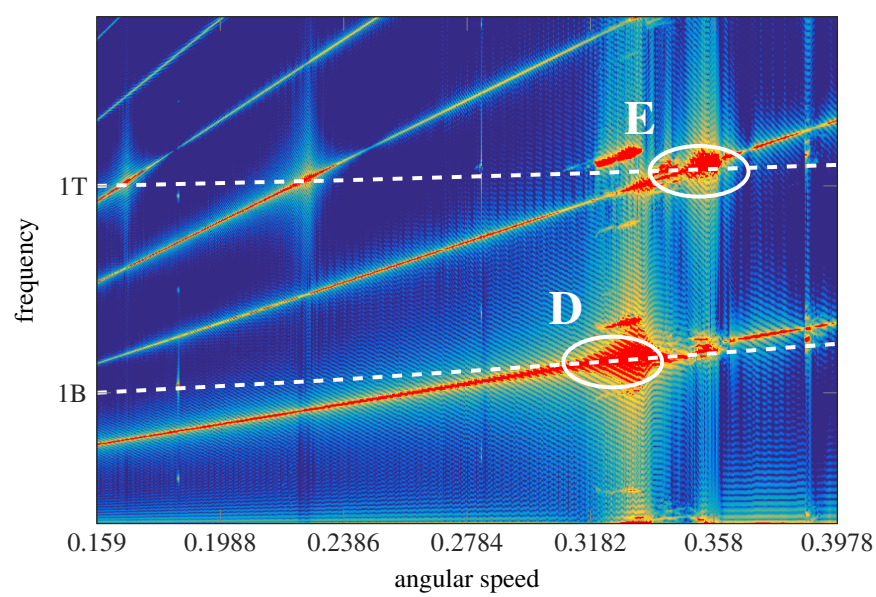

(a) INTIAL PROFILE

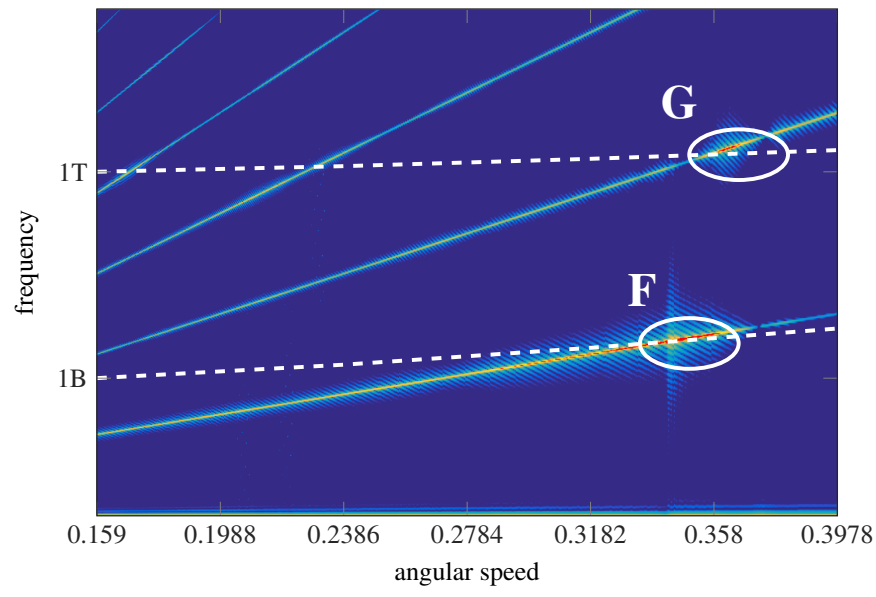

(b) FIRST ITERATED PROFILE

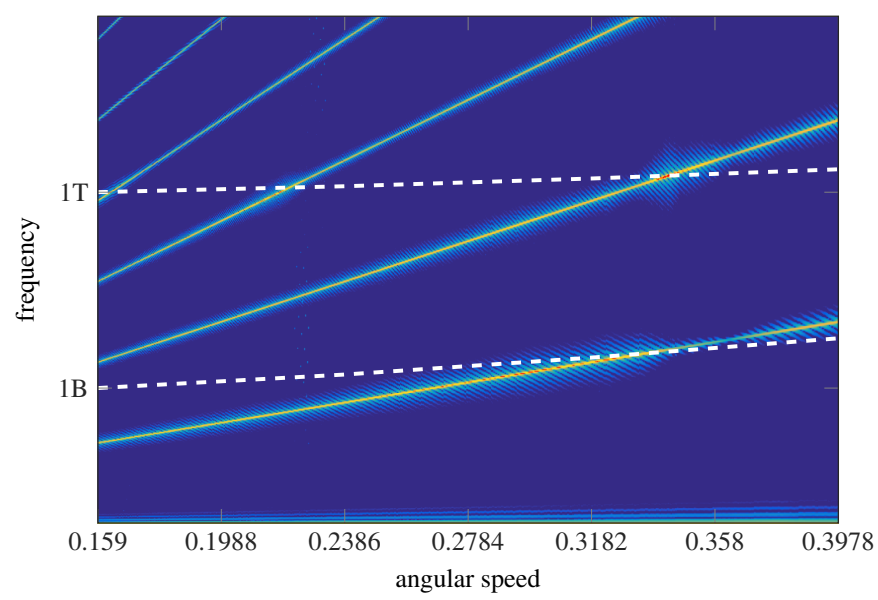

(c) FINAL PROFILE

Figure 4. INTERACTION MAPS WITH 4 LOBES

consumption for each of these profiles.

\section{CLEARANCE CONSUMPTIONS OF ITERATED PROFILES}

Clearance consumptions are presented for the initial profile (Fig. 5), the first iterated profile (Fig. 6) and the final blade profile (Fig. 7) for modes 1B and 1T. 


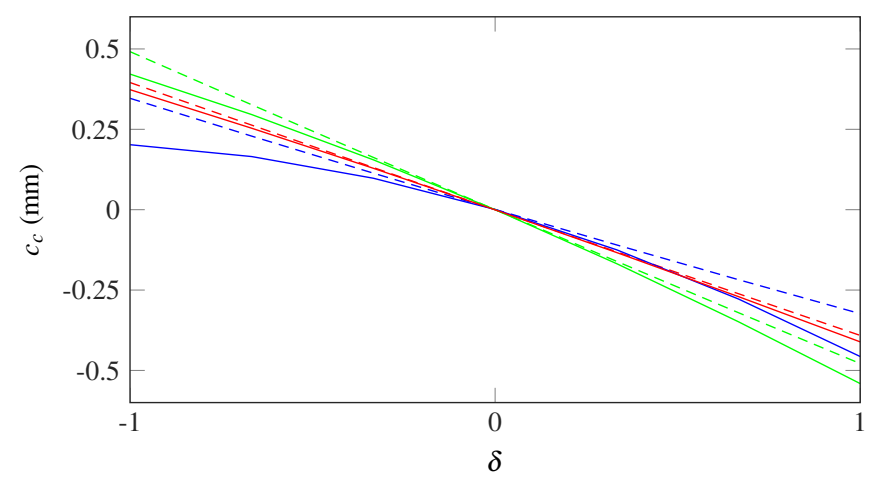

(a) mode $1 \mathrm{~B}$

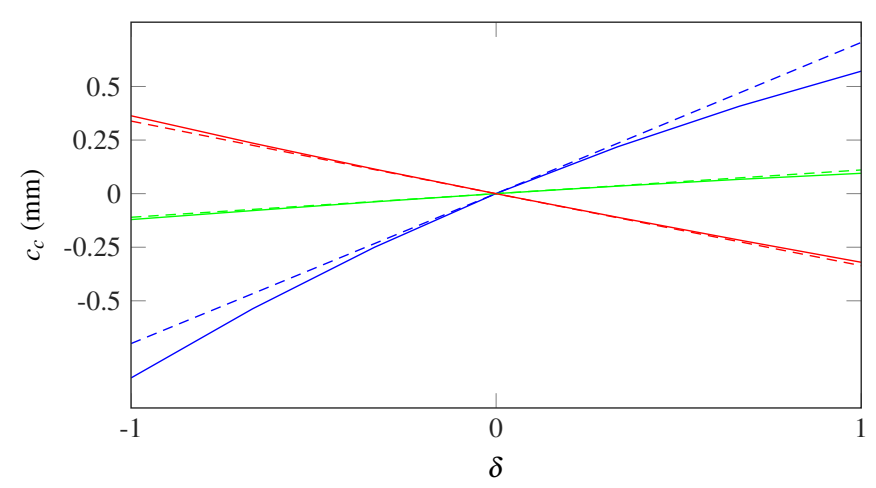

(b) mode $1 \mathrm{~T}$

Figure 5. CLEARANCE CONSUMPTION FOR THE INTIAL PROFILE: LE (linear - - - /nonlinear — - ), MC (linear - - - /non-linear — $)$, and TE (linear - - - /non-linear — $)$

\section{Linear / non-linear models}

Contact simulations carried out in [1] are highly non-linear due to the management of blade-tip/abradable coating and blade-tip/casing contacts. However, since the focus is made on the arisal of interaction phenomena, considering small perturbations provides an acceptable framework for conducting such analysis and advantageously allows for using linear finite element models which yields significant gain in computational times. Thus, the impact of using linear models instead of non-linear finite element models for the computation of clearance consumptions must be assessed: attention must be paid to potential differences between clearance consumptions based on the finite element models used in contact simulations and the actual blade profile. In order to ensure that the obtained results are relevant all clearance consumptions are pictured for both the linear model used in contact simulations (dashed lines - - ) and a non-linear finite element model (plain lines -). Clearance consumptions are computed in a similar fashion for both types of models.

It is noticeable that for all the considered profiles, linear models yield a fairly good approximation of clearance consumptions obtained with the non-linear models, see Figs. 5, 6 and 7. Obviously, largest differences are observed for the largest deformations: in the vicinity of $\delta=-1$ or $\delta=+1$. Interestingly, the linear models of all blade profiles provide an excellent approximation of clearance consumptions for the middle of chord and the trailing edge. Indeed, largest differences are essentially found for the leading edge (---/_- ). Finally, it seems noteworthy to underline that the predicted clearance consumptions of linear models are generally higher than the ones of non-linear models. This is an expected result: indeed, for amplitudes of vibration going beyond the framework of small perturbations, the deformation of linear models will become invalid with a potentially infinite extension of the blade length - yielding an infinite clearance consumption — while the non-linear models will capture the blade contorsion. This observation suggests that for the blade of interest, the employed numerical strategy may exacerbate its sensitivity to contact interactions as it tends to increase blade/casing penetrations. Overall, the good agreement between linear 


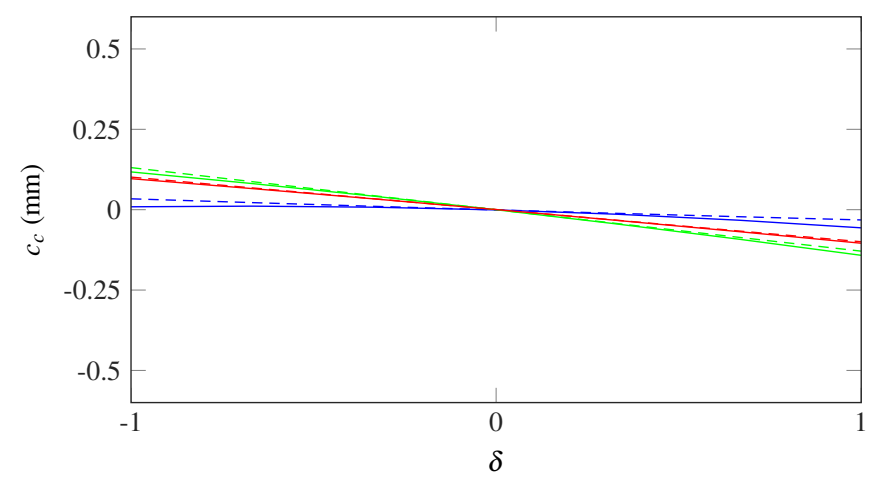

(a) mode $1 \mathrm{~B}$

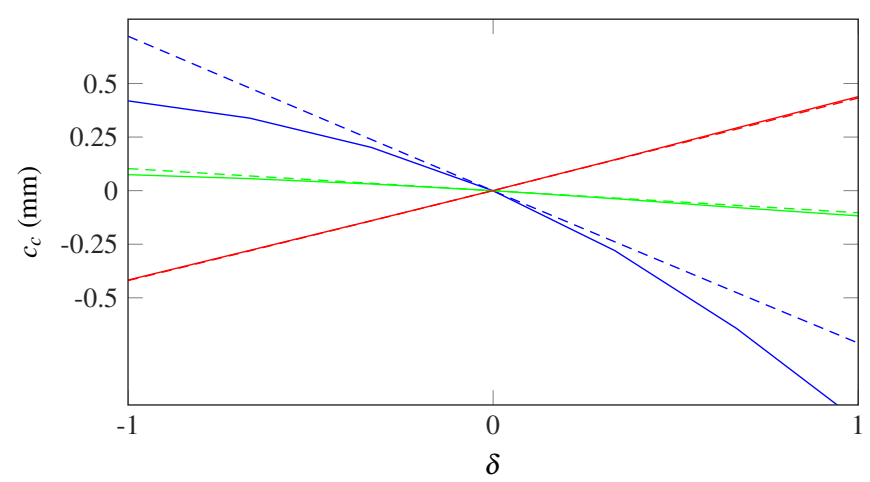

(b) mode $1 \mathrm{~T}$

Figure 6. CLEARANCE CONSUMPTION FOR THE FIRST ITERATED PROFILE: LE (linear - - - /non-linear ——), MC (linear - - - /non-linear — $—$ ), and TE (linear - - - /non-linear $—$ )

and non-linear finite element models justifies a posteriori that linear finite element models be considered for the contact simulations carried out in [1].

\section{Profile comparison}

A close comparison of clearance consumptions obtained for each profiles highlights a few keypoints:

1. first of all, it is patent that clearance consumptions of the initial profile along the first bending mode (1B) are significantly higher than the clearance consumptions predicted for the other profiles. From the leading edge to the trailing edge, clearance consumptions of the initial profile are 5 to 10 times larger than the ones of the final profile, see Figs. 5(a) and 7(a),

2. the order of magnitude of clearance consumptions predicted for the first torsion mode are comparable for all blade profiles and are significantly higher than the ones predicted for the first bending mode. This observation hints that:

(a) in agreement with previous numerical investigations [11], the first torsion mode seems to play a less critical role in the blade dynamics when rubbing occurs compared to the first bending mode,

(b) amplitudes of vibration related to the first torsion mode are generally much lower than the ones of the first bending mode,

3. specifically for mode $1 \mathrm{~T}$, the evolution of clearance consumptions is reversed between the initial profile (Fig. 5(b)) and the other profiles (Figs. 6(b) and 7(b)).

In the end, the computation of clearance consumptions allows for a clear distinction between the intial profile and the final profile. Indeed, as it vibrates along its mode 1B, the amplitude of the clearance consumption for the final profile is negligible. This indicates that the final profile shape is in perfect agreement with its surrounding casing: its vibration along mode $1 \mathrm{~B}$ does not generate any unwanted penetration within the casing. These results suggest that blade dynamics along its first bending mode should be looked at in two directions 


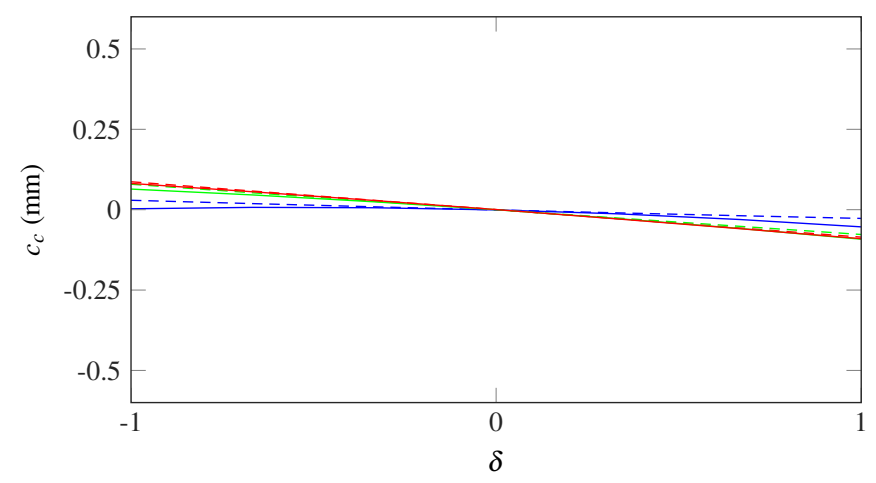

(a) mode $1 \mathrm{~B}$

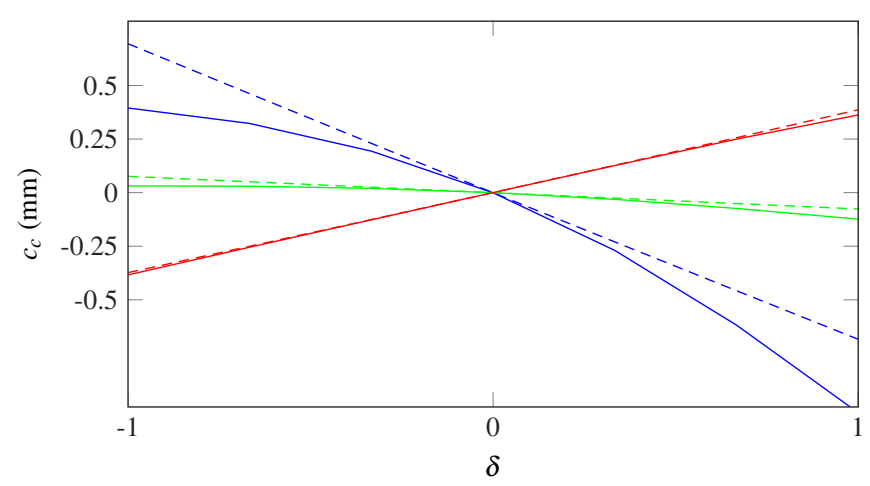

(b) mode $1 \mathrm{~T}$

Figure 7. CLEARANCE CONSUMPTION FOR THE FINAL PROFILE: LE (linear - - - /non-linear $\longrightarrow$ ), MC (linear - - - /non-linear — $)$, and TE (linear - - - /non-linear ——)

$(\delta<0$ and $\delta>0)$. Looking at the blade deformation when contacts occur typically involve only one direction and might lead to inaccurate results for the design of blades robust with respect to contact conditions.

\section{ANALYSIS FOR NON-DIVERGENT INTERACTIONS}

The interaction areas highlighted in Figs. 3(a) and 4(a) lead to the divergence of the numerical simulations. In particular, the amplitudes of vibration may grow to a point that the small perturbation framework is not valid anymore. From a numerical standpoint, it has been observed that the arisal of such interaction is intimately related to the first bending mode of the blade [11]. More recently, the focus has been made on other types of interactions [12] which may involve higher frequency free vibration modes (such as the first torsion mode $1 \mathrm{~T}$ ) and do not yield the divergence of the numerical simulation. Instead, the amplitudes of vibration increase over a few revolutions before contact is lost. Though amplitudes of vibration may be relatively low, such interactions may involve higher frequency free vibration modes and yield crack initiation. The nature of the interaction may be scrutinized through an in-depth post-processing procedure of the acquired time responses (be it from numerical simulations or experimental data) and may be confirmed by the observation of a wear pattern along the abradable coating deposited on the casing.

Such a non-divergent interaction is numerically predicted for the initial profile around the intersection of the first torsional free-vibration mode (1T) and certain high engine orders. The location of this interaction is outside of the angular speed range considered in Fig. 3(a), it appears for lower values of $\Omega$.

Exemplarily, both the initial profile and the final profile are used for the simulation of this non-divergent interaction. A critical speed is found at the intersection of the $22^{\text {nd }}$ engine order with the first torsion mode. Because natural frequencies of both profiles are not perfectly identical, this interaction arises at slightly different angular speeds for each profile. The description of the pre-processing strategy employed for the detection of the 
interaction goes beyond the scope of this article. Results presented in the remainder are given at the critical speed for each profile. The radial displacements of the leading edge of each profile are pictured in Fig. 8. The improvement in terms of amplitude of vibration between the initial profile and the final profile is obvious. Indeed, amplitudes of vibration predicted for the final profile are about seven times lower than the ones predicted for the initial profile. Predicted wear levels on the surrounding abradable coating deposited along the casing contact surface are depicted in Fig. 9. These wear levels are consistent with the aforementioned radial displacements: a much lower wear level is predicted for the final profile. Twenty-two wear lobes are clearly distinguishible for the initial profile $(-)$ in agreement with the fact that the interaction of interest involves the $22^{\text {nd }}$ engine order. For the final profile however, these twenty-two lobes are not obviously visible $(-)$ : the lower amplitudes of vibration did not yield significant wear levels on the casing.

\section{CONCLUSION}

This article focuses on a redesign operation that was carried out on a high-pressure compressor blade in a previous article [1]. The notion of clearance consumption, which may be seen as a quantification of how the blade tends to get closer to the casing as its vibrates along one of its free-vibration mode, allows for a discrimination of blade profiles depending on their sensitivity to rotor/stator interactions initiated by structural contacts. Indeed, blade profiles for which clearance consumption is minimal feature much lower amplitudes of vibration than other blade profiles. Thus, this study suggests that clearance consumption may be a relevant criterion for the design of robust blade profiles with respect to structural interactions.

\section{Acknowledgement}

Thanks go to Snecma for its technical and financial support.

\section{References}

[1] Batailly, A., Legrand, M., Millecamps, A., Cochon, S., and Garcin, F., 2014. "Redesign of a high-pressure compressor blade accounting for nonlinear structural interactions". Journal of Engineering for Gas Turbines and Power, 137, pp. 022502-1-8.

[2] Jones, S., and Legrand, M., 2014. "Forced vibrations of a turbine blade undergoing regularized unilateral contact conditions through the wavelet balance method". International Journal for Numerical Methods in Engineering, 101(5), pp. 351-374.

[3] Zhou, B., Thouverez, F., and Lenoir, D., 2015. "A variable-coefficient harmonic balance method for the prediction of quasi-periodic response in nonlinear systems". Mechanical Systems and Signal Processing, 64-65, pp. 233-244.

[4] Petrov, E., 2015. "Analysis of bifurcations in multiharmonic analysis of nonlinear forced vibrations of gas-turbine engine structures with friction and gaps". In Proceedings of the ASME Turbo Expo 2015 conference, GT2015-43670.

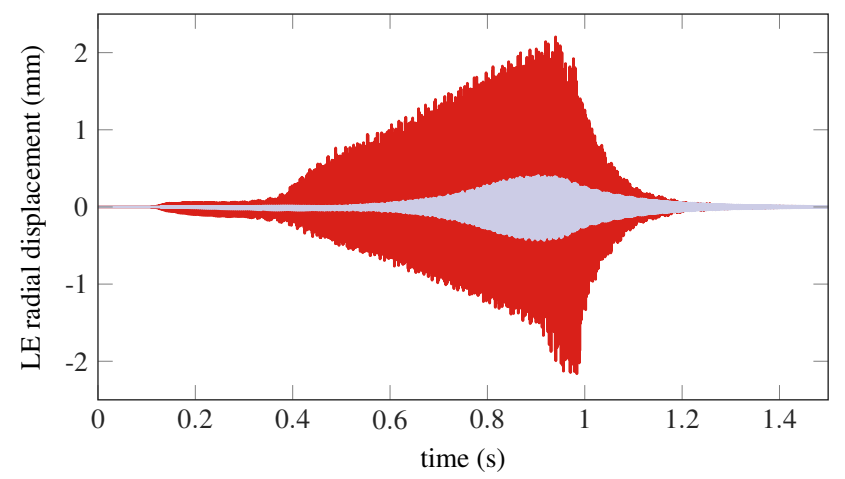

Figure 8. Superimposition of the amplitude increases observed for the initial profile $(-)$ and the final profile $(-)$ 


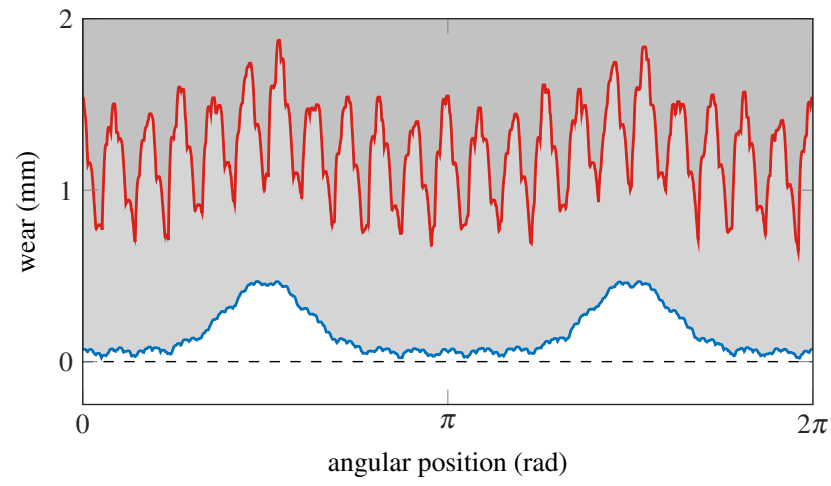

Figure 9. Superimposition of the abradable coating wear levels for the initial profile $(-)$ and the final profile $(-)$. They are compared to the initial abradable coating profile (- - - )

[5] Zhong, J., Han, S., Lu, H., and Kan, X., 2013. "Effect of tip geometry and tip clearance on aerodynamic performance of a linear compressor cascade". Chinese Journal of Aeronautics, 26(3), pp. $583-593$.

[6] Wei, Z., Qiao, W., Shi, P., Chen, P., and Zhao, L., 2014. "Tip-leakage flow loss reduction in a two-stage turbine using axisymmetric-casing contouring". Chinese Journal of Aeronautics, 27(5), pp. 1111 - 1121.

[7] De Maesschalck, c., Lavagnoli, S., Paniagua, G., and Vinha, N., 2014. "Aerothermodynamics of tight rotor tip clearance flows in high-speed unshrouded turbines". Applied Thermal Engineering, 65(1-2), pp. $343-351$.

[8] Erler, E., Vo, H., and Yu, H., 2015. "Desensitization of axial compressor performance and stability to tip clearance size". In Proceedings of the ASME Turbo Expo 2015 conference, GT2015-42746.

[9] Legrand, M., Batailly, A., Magnain, B., Cartraud, P., and Pierre, C., 2012. "Full three-dimensional investigation of structural contact interactions in turbomachines". Journal of Sound and Vibration, 331(11), pp. 2578-2601.

[10] Carpenter, N., Taylor, R., and Katona, M., 1991. "Lagrange constraints for transcient finite element surface contact". International Journal for Numerical Methods in Engineering, 32, pp. 103-128.

[11] Batailly, A., Legrand, M., Millecamps, A., and Garcin, F., 2012. "Numericalexperimental comparison in the simulation of rotor/stator interaction through bladetip/abradable coating contact". Journal of Engineering for Gas Turbines and Power, 134(8), pp. 082504-1-11.

[12] Batailly, A., Legrand, M., Millecamps, A., and Garcin, F., 2015. "Conjectural bifurcation analysis of the contact-induced vibratory response of an aircraft engine blade". Journal of Sound and Vibration, 348, pp. 239-262. 